\title{
Fluorescence Lifetime Imaging of a Caspase-3 Apoptosis Reporter
}

\author{
Johanna M. Buschhaus, ${ }^{1,2}$ Anne E. Gibbons, ${ }^{1}$ Kathryn E. Luker, ${ }^{1}$ \\ and Gary D. Luker ${ }^{1,2,3}$ \\ ${ }^{1}$ Center for Molecular Imaging, Department of Radiology, University of Michigan, Ann \\ Arbor, Michigan \\ ${ }^{2}$ Department of Biomedical Engineering, University of Michigan, Ann Arbor, Michigan \\ ${ }^{3}$ Department of Microbiology and Immunology, University of Michigan, Ann Arbor, \\ Michigan
}

Caspase-3 is a proteolytic enzyme that functions as a key effector in apoptotic cell death. Determining activity of caspase-3 provides critical information about cancer cell viability and response to treatment. To measure apoptosis in intact cells and living mice, a fluorescence imaging reporter that detects caspase-3 activity by Förster resonance energy transfer (FRET) was used. Changes in FRET by fluorescence lifetime imaging microscopy (FLIM) were measured. Unlike FRET measurements based on fluorescence intensity, lifetime measurements are independent of reporter concentration and scattering of light in tissue, making FLIM a robust method for imaging in 3D environments. Apoptosis of breast cancer cells in 2D culture, spheroids, and in vivo murine breast tumor xenografts in response to a variety of genetic and pharmacologic methods implicated in apoptosis of cancer cells was studied. This approach for quantifying apoptosis of cancer cells is based on caspase-3 activity at single-cell resolution using FLIM. ( $) 2017$ by John Wiley \& Sons, Inc.

Keywords: apoptosis $\bullet$ breast cancer $\bullet$ caspase- 3 • fluorescence lifetime imaging $\bullet$ Förster resonance energy transfer

\section{How to cite this article:}

Buschhaus, J. M., Gibbons, A. E., Luker, K. E., \& Luker, G. D. (2017). Fluorescence lifetime imaging of a caspase-3 apoptosis reporter. Current Protocols in Cell Biology, 77, 21.12.1-21.12.12. doi: $10.1002 / \mathrm{cpcb} .36$

\section{INTRODUCTION}

Apoptosis is one form of cell death integral to processes including normal development and treatment of cancer (Ouyang et al., 2012). Many cancer therapies cause cell death through apoptosis, and resistance to apoptosis allows cancer cells to survive and proliferate. Apoptosis proceeds through a series of proteolytic enzymes known as caspases, ultimately activating a central executioner caspase, caspase-3 (Kerr, Wyllie, \& Currie, 1972). Upon activation, caspase- 3 cleaves multiple intracellular proteins at the amino acid sequence aspartate-glutamate-valine-aspartate (DEVD), resulting in apoptotic programmed cell death (Wong, 2011).

Förster resonance energy transfer (FRET) can occur between two spatially close fluorescent proteins with overlap of the emission spectrum of the donor and the excitation spectrum of the acceptor (Suhling et al., 2015). FRET typically is measured by ratios of fluorescence intensities emitted by donor and acceptor proteins following excitation of the donor molecule. Efficient FRET reduces fluorescence from the donor relative to the acceptor. Intensity-based FRET measurements are affected by relative concentrations of 
donor and acceptor molecules, and wavelength-dependent differences in attenuation and scattering of light also distort intensity ratios in tissues (Hoppe, Christensen, \& Swanson, 2002; Shrestha, Jenei, Nagy, Vereb, \& Szöllősi, 2015; Yellen \& Mongeon, 2015). As an alternative to intensity-based measurements, fluorescence lifetime imaging microscopy (FLIM) has been used to quantify FRET. Fluorescence lifetime is an inherent physical property of a fluorescent molecule, representing the time a fluorophore spends in the excited state before returning to the ground state with release of a photon (McGlynn, 1966). FRET interactions shorten the fluorescence lifetime of the donor molecule, which can be detected with quantitative imaging systems. Fluorescence lifetime is unaffected by probe concentration or depth within tissue, overcoming limitations that make intensitybased FRET studies challenging to perform in 3D cell culture settings and animal models.

To quantify apoptosis in systems ranging from intact cells to living mice, FLIM was used to monitor a FRET imaging reporter for caspase-3 (Shcherbakova, Hink, Joosen, Gadella, \& Verkhusha, 2012; Xiao, Gibbons, Luker, \& Luker, 2015). The reporter consists of fluorescent proteins LSSmOrange and mKate2 linked by a consensus DEVD sequence for caspase-3. The long Stokes shift of donor LSSmOrange clearly separates the excitation wavelengths of this protein from the acceptor molecule, mKate2, while still providing enough spectral overlap for FRET. In cells without active caspase-3, the reporter remains intact, shortening the lifetime of LSSmOrange. Cells undergoing apoptosis activate caspase-3, cleaving the imaging reporter and separating the donor and acceptor molecules. Therefore, cells undergoing apoptosis have reduced FRET, which lengthens the lifetime of LSSmOrange.

This unit details steps needed to investigate apoptosis by FLIM in different environments and in response to various treatments. First, two parallel sets of cells are established: one set has the apoptosis reporter, while the second set has only the unfused donor protein (see Basic Protocol 1). Cells with the unfused donor protein, LSSmOrange, serve as controls for the longest lifetime of this protein. Then these cells are used in twodimensional, spheroid, and in vivo models (see Basic Protocol 2). Across model systems, potential genetic and chemical interventions and the imaging method used to measure apoptosis by FLIM are listed (see Basic Protocol 3). Select methods that can be used to validate imaging data for caspase- 3 activation and apoptosis are also described (see Basic Protocol 4).

BASIC PROTOCOL 1

FLIM of Apoptosis 21.12.2

\section{CELL CULTURE AND GENERATION OF STABLE CELL LINES}

In this protocol, human HEK-293T and MDA-MB-231 cells lines are transduced to constitutively express either unfused LSS-mOrange or the caspase-3 apoptosis reporter LSS-mOrange-DEVD-mKate2. However, investigators may use other cell lines and types as appropriate to specific experiments. First, a lentiviral vector of LSS-mOrange pLVX IRES blasticidin and an LSS-mOrange-DEVD-mKate2 vector in a PiggyBac transposon vector are made. The vectors are then introduced into cells to develop cell lines that stably express each reporter. Lastly, cells stably expressing the desired reporter through drug selection (blasticidin) or flow cytometry to establish a relatively uniform population for use in subsequent experiments are identified.

Refer to standard molecular biology texts for general protocols and details on transferring reporters to lentiviral vectors, transiently transfecting cells, stably transducing cells, and selecting for populations of stably-expressing cells.

Maintain cell lines in culture medium according to manufacturer's recommendations. Culture all cell lines discussed in DMEM supplemented with $10 \%$ FBS and $1 \%$ penicillin/streptomycin/glutamine in a $37^{\circ} \mathrm{C}, 5 \% \mathrm{CO}_{2}$ incubator. Passage cells as necessary every 2 to 4 days by trypsinization and resuspension. 
Cell lines:

HEK 293T cells (293T, ATCC ${ }^{\circledR}$ CRL-3216 ${ }^{\text {TM }}$ )

MDA-MB-231 breast cancer cells (231, ATCC ${ }^{\circledR}$ HTB-26 ${ }^{\mathrm{TM}}$ )

Immortalized human bone marrow cell line HS-5 (HS-5, ATCC ${ }^{\circledR}$ CRL-11882 ${ }^{\mathrm{TM}}$ )

Immortalized human bone marrow cell line HS-27a (HS-27a, ATCC ${ }^{\circledR}$ CRL-2496 ${ }^{\mathrm{TM}}$ )

Dulbecco's modified Eagle medium with high glucose and pyruvate (DMEM,

Gibco $^{\circledR}$, cat. no. 11995-065)

Standard fetal bovine serum (FBS, HyClone ${ }^{\mathrm{TM}}$, cat. no. SH300088.03)

100x penicillin/streptomycin/glutamine (Gibco ${ }^{\circledR}$, cat. no. 10378-016)

$0.25 \%$ Trypsin-EDTA (Gibco ${ }^{\circledR}$, cat. no. 25200-056)

$1 \times$ sterile phosphate buffered saline, pH 7.4 (PBS, Gibco ${ }^{\circledR}$, cat. no. 10010-049)

pcDNA $^{\mathrm{TM}} 6 / \mathrm{V} 5$-His A, B, \& C Mammalian Expression Vectors (pcDNA,

Invitrogen ${ }^{\mathrm{TM}}$, cat. no. V220-20)

PCR primers for blasticidin reading frame amplification (IDT ${ }^{\circledR}$ or similar vendor)

5'-GTGGTTTTCCTTTGAAAAACACGATGATAATATGGCCAAGCCTTTG TCTC- $3^{\prime}$

5' - CCAGACGCGTTCAATTAATTAGCCCTCCCACACATAACCAG-3'

Lentiviral vector pLVX IRES puromycin (Clontech, cat. no. 632183)

Fluorescent protein LSS-mOrange (gift of V. Verkhusha, Albert Einstein College of Medicine)

PCR primers for LSS-mOrange amplification (IDT ${ }^{\circledR}$ or similar vendor)

5'-ATGCGCTAGCGCCACCATGGTGAGCAAGGGCGAGGAG-3'

5'-GCATGCGGCCGCTTACTTGTACAGCTCGTCCATGCCGC-3'

Blasticidin S HCl, powder (ThermoFisher Scientific, cat. no. R21001)

Fluorescent protein LSS-mOrange-DEVD-mKate2 (gift of V. Verkhusha, Albert

Einstein College of Medicine)

Super PiggyBac Transposase expression vector (Systems Bioscience, cat. no. PB210PA-1)

$\alpha$-tri-Calcium phosphate (Sigma-Aldrich ${ }^{\circledR}$, cat. no. 50553)

FuGENE $^{\circledR} 6$ Transfection Reagent (Promega, cat. no. E2691)

PB-CMV-MCS-EF1-Puro cDNA cloning and expression vector (Systems

Bioscience, cat. no. PB510B-1)

$37^{\circ} \mathrm{C}, 5 \% \mathrm{CO}_{2}$ incubator

Flow cytometer

Additional reagents and equipment for PCR and DNA restriction enzyme digestion and ligations

\section{Prepare LSS-mOrange}

1. Generate a lentiviral vector with a selection marker for blasticidin by amplifying the blasticidin reading frame from the $\mathrm{pDNA}^{\mathrm{TM}} 6 / \mathrm{V} 5$-His A expression vector using PCR primers for blasticidin reading frame amplification. Remove the puromycin gene cassette by ligating the generated PCR product into the lentiviral pLVX-IRES-puro vector sites BmgB1 and M1uI.

2. Amplify the vector for fluorescent protein LSS-mOrange using the listed PCR primers for LSS-mOrange amplification. Digest the PCR product and ligate it into the generated pLVX IRES blasticidin vector sites NheI and NotI.

3. Transiently transfect 293 T cells to produce lentiviruses for the LSS-mOrange pLVX IRES blasticidin vector.

Fluorescent

Protein Technology 
4. Stably transduce both $293 \mathrm{~T}$ and 231 cell lines with the generated LSS-mOrange pLVX IRES blasticidin lentivirus. Select for the population of stably expressing cells by drug selection with blasticidin.

Dissolve blasticidin according to manufacturer's instructions to a $10 \mathrm{mg} / \mathrm{ml} \mathrm{stock} \mathrm{solution}$ $(1000 \times)$ and use $1 \times$ for cell selection. Optimal concentrations of blasticidin will vary for different types of cells. Aliquots are stable up to 1 year at $-20^{\circ} \mathrm{C}$. Do not subject blasticidin stocks to multiple freeze-thaw cycles.

\section{Prepare LSS-mOrange-DEVD-mKate2}

5. Excise the FRET reporter cassette LSS-mOrange-DEVD-mKate2 at the NheI and NotI sites. Ligate the product into the corresponding NheI and NotI sites of the PiggyBac transposon vector.

6. Transfect $293 \mathrm{~T}$ cells using calcium phosphate and the 231 cell type using FuGENE 6 with the above-generated vector and corresponding transposase plasmid to generate stably-expressing cells.

A transposon was used for transfection and subsequent stable expression of the LSSmOrange-DEVD-mKate 2 reporter instead of a lentivirus as for the LSS-mOrange. Lentiviral transduction frequently results in homologous recombination due to similarity in DNA sequences of LSS-mOrange and mKate2. Transposons do not use recombination for genome integration. Reagents and conditions used for transfection must be optimized based on cell type.

7. Use flow cytometry to sort for the middle $30 \%$ of the population of stably-expressing cells.

Sorting for the middle 30\% of stably-expressing cells generates a population of cells with relatively uniform levels of the fluorescent reporter that still maintains the heterogeneity known for cancer cell lines. In the authors' experience, sorting for cells with intermediate expression of these reporters maintains stable expression over time.

BASIC PROTOCOL 2

FLIM of Apoptosis

21.12.4

\section{EXPERIMENTAL SETUP}

In this protocol, the experimental setups for imaging apoptosis using FLIM in twodimensional cultures, spheroids, and an animal model are described. All studies focus on determining the apoptotic state of cells using the caspase-3 fluorescent reporter. Twodimensional studies include treatment with a plasmid encoding pro-apoptotic protein BAX, drug assays with the MEK inhibitor trametinib, staurosporine, sodium dichloroacetate, and glucose and/or glutamine deprivation. Spheroid models were treated with trametinib or vehicle to observe 3D conditions. Murine models included treatment with trametinib or vehicle with subsequent histology and immunohistochemistry.

NOTE: All protocols using live animals must first be reviewed and approved by an Institutional Animal Care and Use Committee (IACUC) and must follow officially approved procedures for the care and use of laboratory animals.

\section{Additional Materials (also see Basic Protocol 1)}

Plasmid encoding pro-apoptotic protein BAX (gift of S. Galbán, University of Michigan)

Empty plasmid vector

Trametinib (GSK112021, SelleckChem $\odot$, cat. no. S2673)

Staurosporine (Cell Signaling Technology ${ }^{\circledR}$, cat. no. 9953S)

Dimethyl sulfoxide (DMSO, Corning ${ }^{\circledR}$, cat. no. 25-950-CQC)

Dulbecco's modified Eagle's medium without glucose, glutamine, or phenol red $\left(\right.$ Gibco $^{\circledR}$, cat. no. A1443001)

Standard fetal bovine serum (FBS, HyClone ${ }^{\mathrm{TM}}$, cat. no. SH300088.03) 
L-Glutamine, $200 \mathrm{nM}\left(\right.$ Gibco $^{\circledR}$, cat. no. 25030081)

Glucose solution (Gibco ${ }^{\circledR}$, cat. no. A2494001)

Sodium pyruvate, $100 \times\left(\right.$ Gibco $^{\circledR}$, cat. no. 11360-070)

Sodium dichloroacetate (DCA, Sigma-Aldrich ${ }^{\circledR}$, cat. no. 347795)

Dulbecco's modified Eagle's medium with high glucose and without phenol red

(PRF DMEM, Gibco ${ }^{\circledR}$, cat. no. 31053-028)

Penicillin/streptomycin/glutamine, $100 \times\left(\mathrm{P} / \mathrm{S} / \mathrm{G}\right.$, Gibco ${ }^{\circledR}$, cat. no. 10378-016)

$0.9 \%(\mathrm{w} / \mathrm{v}) \mathrm{NaCl}$ solution, sterile

12- to 14-week-old female NSG mice (The Jackson Laboratory)

Carboxymethylcellulose, sodium salt, low-viscosity (Calbiochem, cat. no. 217277)

Tween ${ }^{\circledR} 80$ (Sigma-Aldrich ${ }^{\circledR}$, cat. no. P4780-100ML)

Isoflurane

Depilatory solution (e.g., Nair ${ }^{\mathrm{TM}}$ )

$10 \%$ formalin

Cleaved caspase-3 (Asp175) antibody (Cell Signaling Technology ${ }^{\circledR}$, cat. no. 9661)

$\mathrm{H} \& \mathrm{E}$

6-well plates

384-well, low-volume, black round-bottom polystyrene $\mathrm{NBS}^{\mathrm{TM}}$ microplates, non-sterile (Corning ${ }^{\circledR}$, cat. no. 3676)

UV source

Transfer, imaging, and analysis (TRIM) plates (Cavnar, Salomonsson, Luker,

Luker, \& Takayama, 2014)

Microscope

Small animal shaver (Wahl compact cordless trimmer or similar instrument)

Various surgical instruments

\section{Two-dimensional treatment and imaging studies}

1a. Transiently transfect $293 \mathrm{~T}$ cells expressing LSS-mOrange-DEVD-mKate2 using calcium phosphate with varying concentrations of BAX-encoding plasmid (e.g., 500, 1000, and $2000 \mathrm{ng}$ ). As a control, transfect 293T cells expressing LSS-mOrangeDEVD-mKate2 with the highest concentration of empty vector.

2a. One-day post-transfection, seed 1.4E5 transfected cells into each well of a 6-well plate. Repeat this step for both the above-transfected BAX \pm 293T LSS-mOrangeDEVD-mKate2 cell type and the 231 LSS-mOrange-DEVD-mKate2 cell type.

3a. One day before imaging studies, treat cells as follows:

i. For compound studies, use $100 \mathrm{nM}$ trametinib, $1 \mu \mathrm{M}$ staurosporine, or DMSO vehicle control.

ii. For nutrient-deprivation studies, use DMEM without glucose, glutamine, or phenol red supplemented with $10 \%$ FBS and either $1 \%$ glucose, $1 \%$ glutamine, or neither.

Phenol red is omitted for fluorescence imaging assays to reduce background signal.

iii. For DCA studies, use $40 \mathrm{mM}$ DCA in DMEM without glucose, glutamine, or phenol red supplemented with $10 \%$ FBS, $1 \%$ sodium pyruvate, and either $1 \%$ glucose or $1 \%$ glutamine.

\section{Spheroid model treatment and imaging studies}

1b. Prepare complete PRF DMEM with base PRF DMEM supplemented with $10 \%$ FBS and $1 \% \mathrm{P} / \mathrm{S} / \mathrm{G}$.

2b. Before seeding cells, sterilize 384-well plates with $90 \mathrm{sec}$ of UV radiation. Place 600 LSS-mOrange or LSS-mOrange-DEVD-mKate2 cells expressing 231 cells mixed

Fluorescent Protein Technology 
with 2400 HS-5 or HS-27A cells into each well. This yields a total of 3E3 cells per well with $20 \%$ cancer cells and $80 \%$ stromal cells in $25 \mu$ l of complete PRF DMEM.

Cancer cells were seeded with stromal cells to form viable spheroids. Without supporting stromal cells, not all cancer cell lines and types will form compact spheroids.

3b. One day after seeding, carefully remove $18 \mu \mathrm{l}$ of medium from each well and gently replace it with $20 \mu \mathrm{l}$ complete PRF DMEM containing either $100 \mathrm{nM}$ trametinib or DMSO as its corresponding vehicle control.

4b. Image spheroids $24 \mathrm{hr}$ after treatment by transferring spheroids to a TRIM plate to allow the upright microscope objective to be immersed in liquid while imaging.

The TRIM (transfer, imaging, and analysis) plate was designed to facilitate transfer and stabilization of spheroids for fluorescence microscopy. The protocol for fabricating the TRIM plate has been described previously (Cavnar, Salomonsson, Luker, Luker, \& Takayama, 2014).

\section{Animal models and intravital microscopy}

1c. Suspend MDA-MB-231 cells expressing either LSS-mOrange or LSS-mOrangeDEVD-mKate 2 at a concentration of $5 E 5$ cells $/ 50 \mu l$ in sterile $\mathrm{NaCl}$ solution. Keep cells on ice until use.

2c. Inject $50 \mu 1$ cell-suspension into each fourth inguinal mammary fat pad of 12- to 14-week-old female NSG mice as previously described (Luker et al., 2012). Treat mice daily after 3- to 4-mm diameter tumors form with $50 \mu \mathrm{l}$ oral gavage of either $1 \mathrm{mg} / \mathrm{kg}$ trametinib or appropriate vehicle control.

NSG mice (NOD.Cg-Prkdc scid Il2 $\mathrm{rg}^{\mathrm{tm} / \mathrm{Wjl}} / \mathrm{SzJ}$ ) are a highly immunocompromised strain of mice that allow the growth of human breast cancer xenografts. Alternative strains of immunocompromised mice may be used. However, growth of human breast cancer cells is slower in less immunocompromised mice. Younger mice may be used if desired.

Tumor formation takes $\sim 20$ days, but the time depends on various factors including mouse type, cell type, and numbers of injected cells.

Formulate trametinib for gavage (Kwong et al., 2012) by dissolving in sterile 100\% DMSO and diluting 1:9 in filter-sterilized 1\% carboxymethylcellulose and $0.4 \%$ Tween-80. Formulations for other drugs and compounds will likely differ.

3c. Image tumors both 8 and 14 days after consecutive treatment by intravital microscopy.

\section{Perform histology}

4c. After 14 days of treatment, excise tumor from mouse and fix in $10 \%$ formalin. Embed, slice, and process tissue slides based on standard staining techniques.

5c. Perform immunohistochemistry for the cleaved caspase-3 reporter and histology with $H \& E$.

6c. Evaluate the number of cells positive for the cleaved caspase-3 reporter for comparison to FLIM data.

BASIC PROTOCOL 3

FLIM of Apoptosis

\section{METABOLIC FLUX STUDIES}

This protocol details the use of the Seahorse Bioscience XFe analyzer to carry out a metabolic flux assay. The Glycolysis Stress Test was used to determine effects of various nutrient conditions or treatments on metabolism and these data were related to apoptosis measured with the caspase-3 reporter. 
MDA-MB-231 breast cancer cells (231, ATCC ${ }^{\circledR}$ HTB-26 ${ }^{\mathrm{TM}}$ )

Dulbecco's modified Eagle's medium with high glucose and pyruvate (DMEM, Gibco $^{\circledR}$, cat. no. 11995-065)

Standard fetal bovine serum (FBS, HyClone ${ }^{\mathrm{TM}}$, cat. no. SH300088.03)

Seahorse Bioscience XF calibrant (Agilent, cat. no.100840-000)

$100 \times$ pyruvate $\left(\right.$ Gibco $^{\circledR}$, cat. no. $\left.11360-070\right)$

$100 \times$ glutamine $\left(\right.$ Gibco $^{\circledR}$, cat. no. 25030-081)

$1 \mathrm{M}$ glucose, dissolved in sterile water (Sigma Aldrich, cat. no. G5767)

Seahorse Bioscience XF base medium (Agilent, cat. no. 102353-100)

$0.1 \mathrm{~N} \mathrm{NaOH}$

Seahorse Bioscience Glycolysis Stress Test kit (Agilent, cat. no. 103017-100)

Seahorse Bioscience XF96 96-well cell culture microplate (Agilent, cat. no. 101085-004)

$37^{\circ} \mathrm{C}, 5 \% \mathrm{CO}_{2}$ incubator

$37^{\circ} \mathrm{C}$ incubator

Seahorse XFe96 analyzer

Multichannel pipette

Seahorse Bioscience Wave program

1. On the day before the assay, seed 5000231 cells/well in $80 \mu \mathrm{l} /$ well complete DMEM with 10\% FBS in a Seahorse Bioscience XF96 96-well cell culture microplate.

a. Allow plate to sit in hood for $1 \mathrm{hr}$ after seeding for even cell distribution.

b. Incubate overnight in a $37^{\circ} \mathrm{C}, 5 \% \mathrm{CO}_{2}$ incubator.

2. Hydrate cartridge by adding $200 \mu \mathrm{l} \mathrm{XF}$ calibrant to each well and incubating in a non- $\mathrm{CO}_{2}$ incubator overnight at $37^{\circ} \mathrm{C}$.

3. On the day of the experiment, prepare $100 \mathrm{ml}$ assay medium as follows:

$1 \mathrm{ml} 100 \mathrm{mM}$ pyruvate

$1 \mathrm{ml} 200 \mathrm{mM}$ glutamine

$1 \mathrm{ml} 1 \mathrm{M}$ glucose solution

$100 \mathrm{ml}$ Seahorse Bioscience XF base medium pre-warmed to $37^{\circ} \mathrm{C}$

Adjust $\mathrm{pH}$ to 7.4 with $0.1 \mathrm{~N} \mathrm{NaOH}$

Filter sterilize using a $22-\mu \mathrm{m}$ filter and keep at $37^{\circ} \mathrm{C}$

4. On the day of the experiment, change medium in the cell culture plate by first removing $60 \mu \mathrm{l}$ of medium from each well. Take care not to touch the bottom of the well as the action may dislocate cells. Next, add and subsequently remove $180 \mu \mathrm{l}$ Seahorse assay medium to and from each well of the plate two times. Finally, add a final volume of $180 \mu \mathrm{l}$ assay medium to each well.

5. Incubate cell culture plate $1 \mathrm{hr}$ in a non- $\mathrm{CO}_{2}$ incubator at $37^{\circ} \mathrm{C}$ before inserting into the XF analyzer.

6. Resuspend the glucose, oligomycin, and 2-deoxyglucose from the Glycolysis Stress Test kit in assay medium per manufacturer's instructions.

7. Immediately prior to beginning the experiment, inject $10 \mathrm{mM}$ glucose, $2 \mathrm{mM}$ oligomycin, and $100 \mathrm{mM}$ 2-deoxyglucose to Ports A, B, and C of the cartridge, respectively, with a multichannel pipette.

8. Insert the cartridge into the analyzer and follow manufaturer's instructions before inserting cell culture plate.

9. Indicate the injection and measurement strategy on the XF analyzer and perform the experiment by following prompts from the software.

Fluorescent

Protein Technology 
10. Analyze the data using the Wave program provided by Seahorse Bioscience.

$B A S I C$ PROTOCOL 4

\section{FLIM AND DATA ANALYSIS}

The steps for imaging the experimental setups described in Basic Protocol 2 using FLIM are outlined here. The aim of FLIM is to gather data on the caspase-3 fluorescent reporter and apoptosis by taking advantage of the FRET pair LSS-mOrange and mKate2. The FLIM imaging protocol is applicable to the described two-dimensional, spheroid, and murine tumor conditions.

\section{Materials}

2-Photon upright imaging system with variable laser power and compatible $25 \times$ objective equipped with an $80 \mathrm{MHz}$ pulsed scanning laser (Spectra Physics Mai Tai-Deep Sea or comparable product) and 572/15-nm emission filter

Frequency domain instrument for FLIM (FastFLIM, ISS or comparable product) VistaVision Software or comparable product for data analysis (ISS)

1. Keep laser on for at least $30 \mathrm{~min}$ before calibrating the FLIM imaging system as per manufacturer's instructions.

An upright Olympus FVE1000 MPE microscope was used for all 2-photon microscopy. A $25 \times$ NIR-corrected objective (XLPLN25XWMP, NA $=1.05$, Olympus) was used in conjunction with the microscope.

2. After the objective is liquid immersed either in the 2D dish, TRIM plate, or above a tumor, move the field of view to an appropriate imaging plane and location. Image orthotopic mammary tumors as previously described (Salomonnson, Mihalko, Verkhusha, Luker, \& Luker, 2012).

3. Excite the LSS-mOrange protein at $820 \mathrm{~nm}$ using an $80-\mathrm{MHz}$ pulsed scanning laser. Capture emission data through a 572/15 nm emission filter and in the frequency domain using a frequency domain instrument.

A $256 \times 256$-pixel image with a 100- $\mu$ sec dwell time over 15 to 30 frames is sufficient for counts and data acquisition.

Long Stokes Shift (LSS)-mOrange is a fluorescent protein with an excitation wavelength of $820 \mathrm{~nm}$ that emits in the orange channel $(572 \mathrm{~nm})$. mKate 2 excites at either $760 \mathrm{~nm}$ or $1040 \mathrm{~nm}$ and is therefore not excited by the 820-nm excitation wavelength, reducing crosstalk between the two proteins.

4. Use VistaVision or similar image processing software to analyze fluorescence lifetime data as follows.

a. Open acquired images with the Multi-Image Phasor Analysis function.

b. Set the Gaussian smoothing operation to 3 and the minimum threshold count to 10 to eliminate noise

The Gaussian instead of the median smoothing operation is used because it takes into account lifetimes of surrounding pixels. A value of 3 for the operation smoothes the image while maintaining spatial accuracy. The minimum threshold count may vary between experiments as cell type, environment, and other factors may add noise. Change the minimum threshold count to minimize the number of pixels in the background within ROIs while not sacrificing cell data.

c. Select the population of cells with a longer lifetime from the phasor plot with a red ROI to define the portion of cells with less caspase-3 activity. Select the population of cells with a shorter lifetime with a yellow ROI to define the cells with increased caspase- 3 activity and subsequent apoptosis. 


\section{COMMENTARY}

\section{Background Information}

Fluorescence lifetime imaging (FLIM) is a powerful imaging modality that extracts information from fluorescent proteins about parameters such as environment (i.e., $\mathrm{pH}$ ) and interactions. Protein conformational changes, association, and/or separation are identifiable by Förster resonance energy transfer (FRET), changing the fluorescence lifetime of the donor fluorophore (Suhling et al., 2015). FRET measured by FLIM can detect both inter- and intracellular interactions on a single-cell basis (Jares-Erijman \& Jovin, 2003). Unlike FRETimaging-based on ratios of fluorescence intensity from donor and acceptor fluorophores, FLIM data are unaffected by greater absorption of shorter versus longer wavelengths of light by tissue. This advantage of FLIM is particularly critical for imaging through depth in 3D cell culture models, such as spheroids and animals. The LSS-mOrange and mKate2 FRET pair developed by Shcherbakova et al. also provides advantages over the standard pair of cyan and yellow proteins because longer wavelengths of light penetrate better through tissues, improving imaging depth.

Alternative methods for studying apoptosis include ratiometric FRET imaging (discussed above) cell counting, bioluminescence imaging (Wang et al., 2016), and dyes that differentially stain living versus dead cells. Cell counting is most effective in tightly controlled in vitro environments and often requires removing cells from their experimental environments. Bioluminescence imaging provides population-scale measurements and requires adding a substrate to the environment, producing time-dependent changes in signal depending on kinetics of the luminescence reaction. Several dyes have been used to visualize apoptosis at a single-cell scale (Bouchier-Hayes, Muñoz-Pinedo, Connell, \& Green, 2008). Dyes, however, dilute with cell divisions, depend on fluorescence intensities affected by depth in tissue, and potentially vary based on pharmacokinetics of delivery in vivo. FLIM imaging allows apoptotic cells to be imaged at single-cell resolution without the need for exogenous substrates.

Fast frequency domain FLIM (FFD) is used for imaging studies. FFD extracts data from phase delay and modulation ratio of excitation and emission light and has the advantage of real-time imaging of changing systems, such as apoptosis. Unlike population-scale metrics of apoptosis, single-cell imaging detects heterogeneity of responses among cancer cells in complex 3D and in vivo environments. Heterogeneity of responses may identify emergence or selection of drug resistant populations from cell autonomous or stromal-dependent mechanisms. Importantly, FLIM captures both spatial and temporal dynamics of these responses to different stimuli. Overall, the imaging method described offers new opportunities to investigate apoptosis at single-cell resolution in living systems, advancing knowledge of tumor heterogeneity that ultimately may help overcome drug resistance in cancer.

\section{Critical Parameters}

It is critical that the FLIM unit be calibrated to a known fluorescent lifetime before beginning any imaging. This must be done at least 30 min after turning on and tuning the laser to the desired imaging wavelength, allowing sufficient time for the system to stabilize. FLIM imaging is extremely sensitive to any ambient light, so the imaging stage should be as lightinsulated as possible. Any non-critical light in the room should be turned off to avoid skewing data.

To reduce background signal and increase sample data accuracy, performing a background correction is recommended. This may be done by imaging a sample of the medium used in the specific experimental setup and running the background correction function in the FLIM data processing software. It is also advised to keep scan parameters (i.e., image size, pixel dwell time, laser intensity, and scan count) consistent during an experiment. This allows for more consistent analysis and results and decreases the chance of needing to alter the minimum count threshold between images.

FLIM imaging in living animals may be degraded by respiratory motion, reducing resolution of images and quality of data. Therefore, properly immobilizing a tumor or other tissue of interest is necessary for optimal results. Typically, investigators use a combination of surgery to expose the desired imaging site and holder devices to restrain the tissue during imaging while maintaining appropriate vasculature and perfusion (Masedunskas et al., 2012).

\section{Anticipated Results}

Basic Protocol 1 yields two matching pairs of 293T and MDA-MB-231 cell types, the first expressing the caspase- 3 apoptosis reporter, while the second expresses only the
Fluorescent Protein Technology 

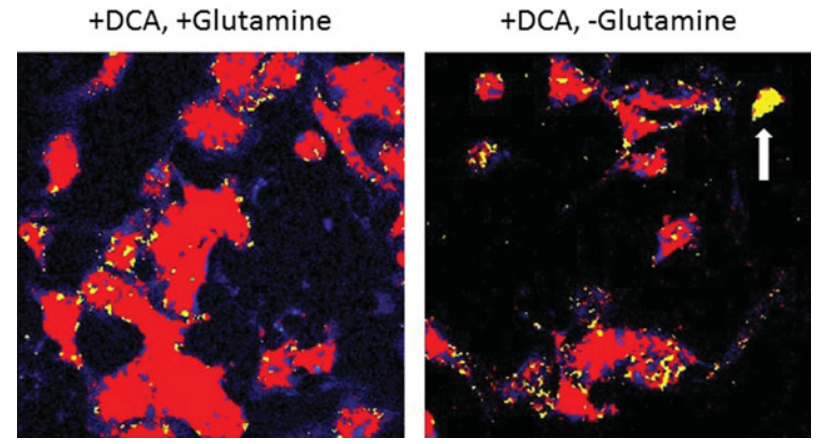

Figure 21.12.1 Representative data of caspase-3 activity of DCA-treated cells in 2D culture with and without glutamine. Image shows FLIM image pseudo-colored by fluorescence lifetime. Yellow pixels correspond to a shorter lifetime indicating cleavage of the DEVD sequence and apoptosis. Red pixels indicate a longer lifetime and greater interaction of the FRET pair. MDAMB-231 cells were treated with DCA to decrease the rate of glycolysis within the cells. Cells in glutamine-containing medium showed minimal caspase-3 activity (increased proportion of red versus yellow pixels). Cells in glutamine-deprived medium showed increased caspase-3 activity (increased proportion of yellow versus red pixels). White arrow shows a cell clearly undergoing apoptosis as displayed by FLIM. Blue pixels represent background fluorescence.
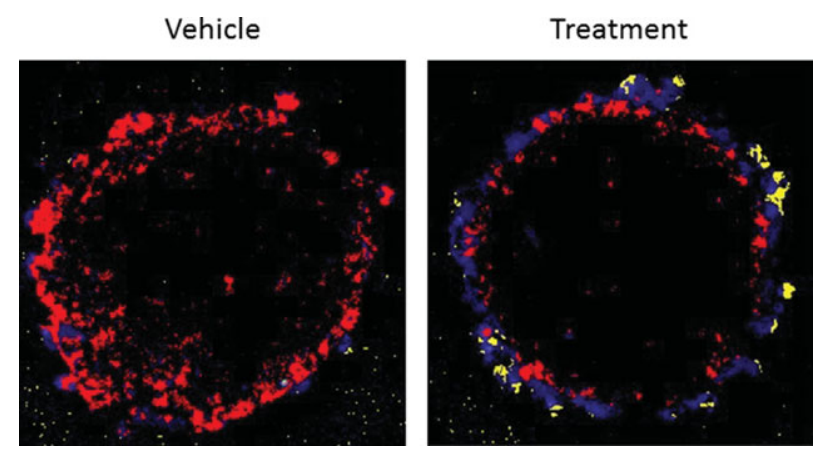

Figure 21.12.2 FLIM images of HS-5 bone marrow stromal cells cultured with MDA-MB-231 breast cancer cells to form 3D spheroids. Spheroids pseudo-colored by fluorescence lifetime with yellow pixels indicating caspase-3 activity and red pixels indicating greater FRET from the intact reporter. The spheroid treated only with vehicle control showed minimal cleavage of the DEVD sequence and apoptotic signal. The spheroid treated with trametinib displayed increased caspase3 activity and apoptosis. Apoptosis is most apparent on the periphery of the spheroid, indicating a differential spatial response of this treatment in a 3D spheroid environment.

unfused donor protein. The unfused donor protein LSSmOrange serves as a reference for the longest possible lifetime of the fused reporter protein. Basic Protocol 2 generates three different experimental models for the caspase-3 reporter cell line. First, a twodimensional cell culture model is described. This model is useful in validating reporter activity of the generated cell lines and reporter responses to drug treatment and nutrient deprivation (Fig. 21.12.1). The spheroid model allows analysis of drug treatments and microenvironmental changes in a simulated, controlled 3D setting (Fig. 21.12.2). Orthotopic mouse xenografts demonstrate the in vivo applications of single-cell apoptosis reporting (Fig. 21.12.3).

Basic Protocol 3 describes methods used to perform a metabolic flux assay using the Seahorse Bioscience XFe analyzer (Fig. 21.12.4). Metabolic flux data can be related to the nutrient deprivation study shown in Figure 21.12.1. FLIM data show less caspase3 activity in cells treated with DCA and glutamine than those treated with DCA without added glutamine. Metabolic flux data show that DCA-treated cells supplemented with glutamine show increased rates of both oxygen 

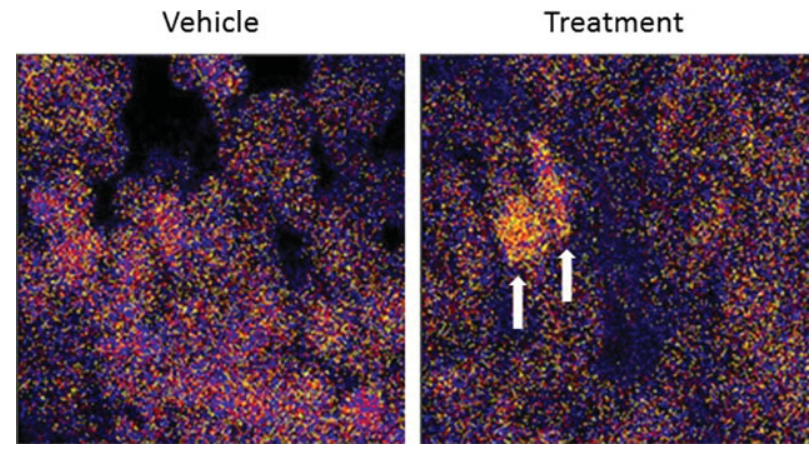

Figure 21.12.3 Representative in vivo FLIM images of orthotopic xenografts of MDA-MB-231 cells. Mice were treated with either vehicle control or the MEK inhibitor trametinib. Image shows FLIM image pseudo-colored by fluorescence lifetime. Yellow pixels correspond to a shorter lifetime indicating cleavage of the DEVD sequence and apoptosis. Red pixels indicate a longer lifetime from the intact caspase-3 FRET reporter. The vehicle mouse tumor has a higher proportion of red than yellow pixels with no large areas of yellow pixels, indicating a longer average lifetime and less apoptosis in this tumor. The trametinib mouse tumor has a higher proportion of yellow than red pixels indicating a shorter average lifetime, increased caspase- 3 activity, and apoptosis. The large area of yellow pixels indicates two apoptotic cells (white arrows).

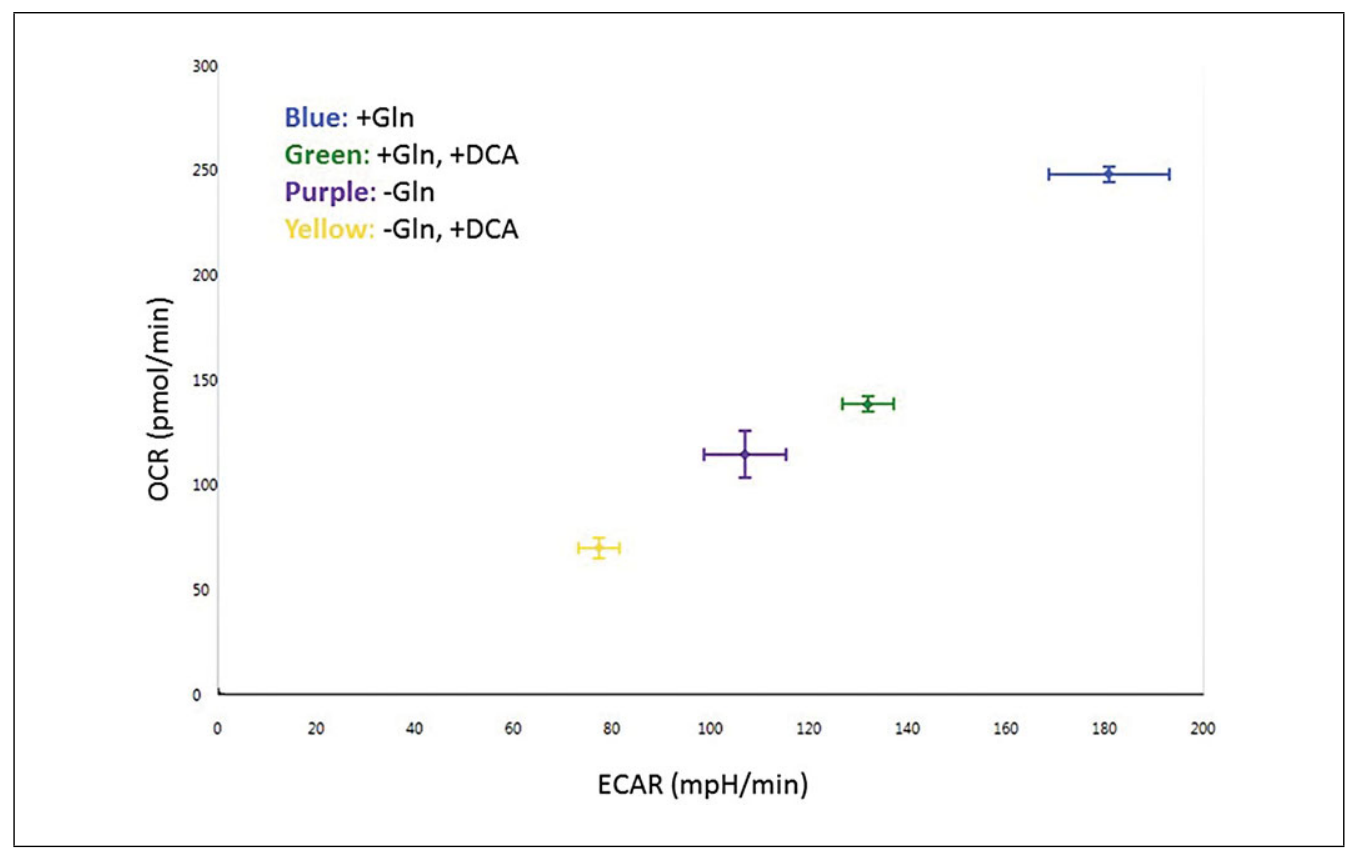

Figure 21.12.4 Energy plot from Seahorse metabolic flux data. MDA-MB-231 reporter cells were incubated under conditions of base DMEM with $1 \%$ glutamine (Gln) only, $1 \%$ glucose only (no Gln), or with $40 \mathrm{mM} \mathrm{DCA}$. The $y$-axis shows the oxygen consumption rate (OCR) and the $x$-axis shows the extracellular acidification rate (ECAR). These are measures of oxidative phosphorylation and glycolysis, respectively.

consumption and extracellular acidification than those cells deprived of glutamine. The FLIM data measuring the increase of caspase3 activity and apoptosis in glutamine-deprived cells are validated by the metabolic flux data, indicating that glutamine-deprived cells are less metabolically active and more susceptible to apoptotic cell death.

\section{Time Considerations}

Basic Protocol 1 requires $\sim 3$ weeks to generate stable cells. Basic Protocol 2 takes $2 \mathrm{hr}$ over 2 days for 2D and spheroid models and $\sim 34$ days for the animal model. Basic Protocol 3 requires 3 hr over 2 days. Tumor imaging studies in Basic Protocol 4 require $\sim 2 \mathrm{hr}$ per mouse.
Fluorescent Protein Technology 


\section{Acknowledgement}

Research supported by NIH grants R01CA195655, R01CA170198, and R01CA196018.

\section{Literature Cited}

Bouchier-Hayes, L., Muñoz-Pinedo, C., Connell, S., \& Green, D. R. (2008). Measuring apoptosis at the single cell level. Methods, 44(3), 222-228. doi: 10.1016/j.ymeth.2007.11.007.

Cavnar, S., Salomonsson, E., Luker, K., Luker, G., \& Takayama, S. (2014). Transfer, imaging, and analysis plate for facile handling of 384 hanging drop 3D tissue spheroids. Journal of Laboratory Automation, 19(2), 208-214. doi: $10.1177 / 2211068213504296$.

Hoppe, A., Christensen, K., \& Swanson, J. A. (2002). Fluorescence resonance energy transfer-based stoichiometry in living cells. Biophysical Journal, 83(6), 3652-3664. doi: 10.1016/S0006-3495(02)75365-4.

Jares-Erijman, E. A., \& Jovin, T. M. (2003). FRET imaging. Nature Biotechnology, 21(11), 13871395. doi: 10.1038/nbt896.

Kerr, J. F. R., Wyllie, A. H., \& Currie, A. R. (1972). Apoptosis: A basic biological phenomenon with wide-ranging implications in tissue kinetics. British Journal of Cancer, 26(4), 239-257. doi: 10.1038/bjc.1972.33.

Kwong, L., Costello, J., Liu, H., Jiang, S., Helms, T., Langsdorf, A., ... Chin, L. (2012). Oncogenic NRAS signaling differentially regulates survival and proliferation in melanoma. Nature Medicine, 18(10), 1503-1510. doi: 10.1038/nm.2941.

Luker, K. E., Mihalko, L. A., Schmidt, B. T., Lewin, S. A., Ray, P., Shcherbo, D., ... Luker, G. D. (2012). In vivo imaging of ligand receptor binding with Gaussia luciferase complementation. Nature Medicine, 18(1), 172-177. doi: 10.1038/nm.2590.

Masedunskas, A., Milberg, O., Porat-Shliom, N., Sramkova, M., Wigand, T., Amornphimoltham, P., \& Weigert, R. (2012). Intravital microscopy: A practical guide on imaging intracellular structures in live animals. Bioarchitecture, 2(5), 143157. doi: 10.4161/bioa.21758.

McGlynn, S. P. (1966). Fluorescence and phosphorescence analysis. Principles and applications.
Journal of the American Chemical Society, 88(23), 5688-5688. doi: 10.1021/ja00975a083.

Ouyang, L., Shi, Z., Zhao, S., Wang, F., Zhou, T., Liu, B., \& Bao, J. (2012). Programmed cell death pathways in cancer: A review of apoptosis, autophagy and programmed necrosis. Cell Proliferation, 45(6), 487-498. doi: 10.1111/j.1365-2184.2012.00845.x.

Salomonnson, E., Mihalko, L., Verkhusha, V., Luker, K., \& Luker, G. (2012). Cell-based and in vivo spectral analysis of fluorescent proteins for multiphoton microscopy. Journal of Biomedical Optics, 17(9), 96001. doi: 10.1117/1.JBO.17.9.096001.

Shcherbakova, D., Hink, M., Joosen, L., Gadella, T., \& Verkhusha, V. (2012). An orange fluorescent protein with a large Stokes shift for singleexcitation multicolor FCCS and FRET imaging. Journal of the American Chemical Society, 134(18), 7913-7923. doi: 10.1021/ja3018972.

Shrestha, D., Jenei, A., Nagy, P., Vereb, G., \& Szöllősi, J. (2015). Understanding FRET as a research tool for cellular studies. International Journal of Molecular Sciences, 16(4), 67186756. doi: 10.3390/ijms16046718.

Suhling, K., Hirvonen, L. M., Levitt, J. A., Chung, P.-H., Tregidgo, C., Le Marois, A., ... Krstajic, N. (2015). Fluorescence lifetime imaging (FLIM): Basic concepts and some recent developments. Medical Photonics, 27, 3-40. doi: https://doi.org/10.1016/j.medpho.2014.12.001.

Wang, Y., Zhang, B., Liu, W., Dai, Y., Shi, Y., Zeng, Q., \& Wang, F. (2016). Noninvasive bioluminescence imaging of the dynamics of sanguinarine induced apoptosis via activation of reactive oxygen species. Oncotarget, 7(16), 22355-22367. doi: 10.18632/oncotarget.7971.

Wong, R. (2011). Apoptosis in cancer: From pathogenesis to treatment. Journal of Experimental \& Clinical Cancer Research, 30, 87. doi: 10.1186/1756-9966-30-87.

Xiao, A., Gibbons, A., Luker, K., \& Luker, G. (2015). Fluorescence lifetime imaging of apoptosis. Tomography, 1(2), 115-124. doi: 10.18383/j.tom.2015.00163.

Yellen, G., \& Mongeon, R. (2015). Quantitative two-photon imaging of fluorescent biosensors. Current Opinion in Chemical Biology, 27, 2430. doi: 10.1016/j.cbpa.2015.05.024. 